\title{
Surface EMG Network Analysis and Robotic Arm Control Implementation
}

\author{
Kwang Ryol Ryu, Member, KIICE
}

\begin{abstract}
An implementation for surface EMG network analysis and vertical control system of robotic arm is presented in this paper. The transmembranes are simulated by equivalent circuit and cable equation for propagation to be converted to circuit networks. The implementation is realized to be derived from the detecting EMG signal from 3 electrodes, and EMG transmembrane signals of human arm muscles are detected by several surface electrodes, high performance amplifier and filtering, converting analog to digital data and driving a servomotor for spontaneous robotic arm. The system is experimented by monitoring multiple steps vertical control angles corresponding to biceps muscle movement. The experimental results are that the vertical moving control level is measured to around 2 degrees and mean error ranges are lower $5 \%$.
\end{abstract}

Index Terms - Surface EMG, electrode, robotic arm, transmembrane, cable equation, Servomotor control and equivalent circuit.

\section{INTRODUCTION}

IT is important to study transmembranes along the membrane of nervous system in biomedical engineering. The neurotransmitters are to convert human's thought, consciousness and external stimulus to ionic electric signals over the afferent neuron, interneuron and efferent neuron of the excited tissues. These researches result in EMG (Electromyography), EEG (Electroencephalogram), ECG (Electrocardiogram), EOG (Electrooculography) and ERG (Electroretinography).[1-3] These systems measure heart, muscle, and brain activity over time by measuring electric potentials on the surface of living tissue. There are clinical and prosthetic controls in EMG signal processing. The signal processing has been studying the control of arm prosthesis. Common applications of the EMG signal are to determine the activation timing of the muscle when the excitation to the muscle begins and ends, to estimate the force produced by the muscle, and to obtain an index of the rate at which a muscle fatigues through the analysis of the frequency spectrum of the signal. Nervous stimuli and muscle contractions can be detected by measuring the ionic current flow in the body. This is accomplished using a biopotential electrode. There

\footnotetext{
Manuscript received October 14, 2011; revised November 8, 2011; accepted November 15, 2011.

Kwng Ryol Ryu is with the Department of Electronic Engineering, Mokwon University, Daejeon, 302-729, S. Korea (Email: conan@mwu.ac.kr)
}

are surface and needle type of EMG electrodes. It is more comfortable and convenient to utilize the surface in acquiring signals in safety than the needle. They are used in medical area and industrial robots. That is, EMG has been applying to human interface system for the handicapped, disability in biomedical electronics and industrial systems. The signals acquired by electrode sensor have been used for the hand gestures and movements, and implemented in the position control of prosthetic arm for amputees and applied to rehabilitation with robotic devices. The position control estimates the intended torque of the stepping motor and provides sufficient information for an effective force control of the arm handicapped as well.[4-7] The system is required to control robotic arm movement in detail according to neurotransmitter signals of arm movement for user's safety spontaneously.

This study is focused on the implementation for vertical robotic arm control system with fine angles. The implementation will be processed by the neurotransmitter signal sensing, amplifier and filtering design, signal processing and system experiment. The sensor has several electrodes, the amplifier and filtering is high performance and low noise and HPF/LPF. The signal processing reveals frequency by Fourier transform, and system performance is measured by relation between EMG signals and servomotor through the iterative experiments.

\section{EMG NETWORK ANALYSIS AND ROBOTIC ARM CONTROL}

\section{A. EMG Network Analysis}

EMG signal of nervous system is required a stimulus to convert to electric system in operating the neuron over the membranes. The signal results from excitable tissue. The travel of muscle via axon is directed from the cranial and spinal cord to a muscle. Interneurons and efferent neurons over the axon are cells that they respond to the chemical transmitter by generating the burst of APs (action potential). The axon is a poor conductor severely attenuated. AP is voltage spikes $100 \mathrm{mV}$ high and a graded potential. The minimum interval of the AP train is $4 \mathrm{~ms}$, refractory period. The AP of the efferent neuron from the interneuron is able to be converted to RLC equivalent network as shown in Fig. 1 to find the electric characteristics. The equivalent circuit is composed of the interneuron input potential $v_{i}$, RLC passive elements and 
the graded potential output $v_{o}$. The inductance of nerve fiber can be neglected. It is swamped out by axoplasmic resistance.

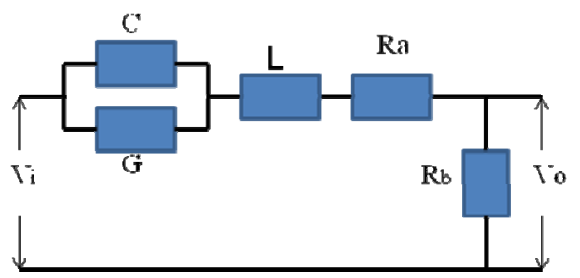

Fig. 1. RLC network of AP.

The transfer function $\mathrm{H}$ of this linear network is defined as output/input applying the Laplace transformation, $\mathrm{H}=\mathrm{Vo}(\mathrm{s}) / \mathrm{Vi}(\mathrm{s})$. The corresponding input potential for AP is given by equation (1), where $\mathrm{k}$ is final value after adaptation, $\tau_{1}$ is time constant of the leading edge, and $\tau_{2}$ is adaptation time constant.

$$
V_{o}(s)=V_{i}\left[k / s=1 /\left(s+1 / \tau_{1}\right)+(1-k) /\left(s+1 / \tau_{2}\right)\right.
$$

For the preceding numerical example, $\mathrm{Vi}(\mathrm{s})=0.02 / \mathrm{s}, \mathrm{k}=0.1$ $\tau_{1}=0.8 \mathrm{~s}, \tau_{2}=2.5 \mathrm{~s}, \mathrm{H}$ is given by equation (2), and the simulation is shown in the Fig. 2. The simulation is given in $\mathrm{c}=0.89, \mathrm{~b}_{1}=0.4, \mathrm{a}_{1}=1.25$ and $\mathrm{a}_{2}=0.045$ for instance.

$$
H(s)=\frac{c\left(s+b_{1}\right)}{\left(s+a_{1}\right)\left(s+a_{2}\right)}
$$

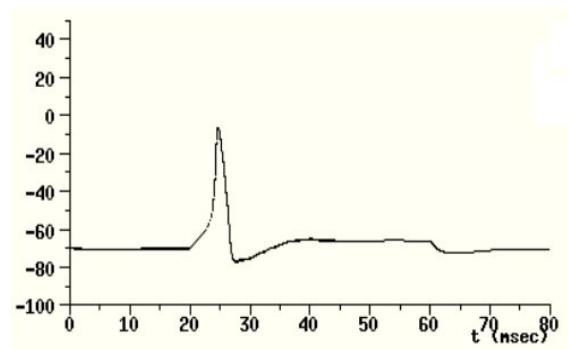

Fig. 2. Simulated AP output waveform.

The transmembrane cable equation derives from Hodgkin and Huxley action potential used the equivalent circuit of Fig. 3 for the short length of an axon. Gn is the equivalent conductance of the membrane to sodium ions, $\mathrm{G}_{\mathrm{k}}$ is the conductance to potassium ions, $\mathrm{Gc}$ is the nominal conductance to chloride ions, and these total membrane resistance is $R_{m}[\Omega . m] . C_{m}[F / m]$ is a membrane capacitance.

The voltage sources are equivalent values at rest. En small sodium ion current flows into the axoplasm, Ek small potassium current flows out of the axoplasm, and $E_{c}$ chloride ions flow out of the axoplam. $R_{i}$ and $\operatorname{Re}[\Omega / \mathrm{m}]$ are denoted by the intracellular and extracellular resistances per length along the fiber.

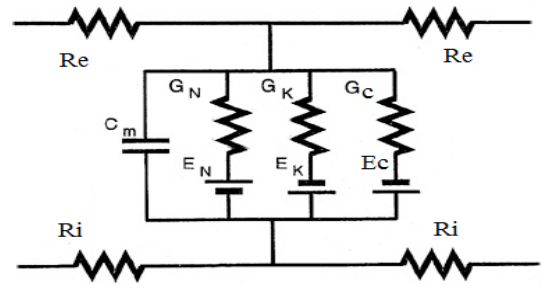

Fig. 3. Transmembrane equivalent network.

The standard cable analysis can be used to derive the cable equation for the transmembrane potential $\mathrm{Vm}$ as defined by equation (3), where $\tau$ is the time constant $\mathrm{RmCm}$, and $\mathrm{L}$ is the length constant $\left(\mathrm{Rm} /\left(\mathrm{Ri}+\mathrm{Re}_{\mathrm{e}}\right)\right)^{2}$.

$$
L^{2} \frac{\partial^{2} V_{m}}{\partial x^{2}}=\tau \frac{\partial V_{m}}{\partial t}+V_{m}
$$

To approximation, a fiber can be represented as the infinitely long $\mathrm{RC}$ cable where $\mathrm{R}$ is series axoplasm resistance and $\mathrm{C}$ is shunt membrane capacitance from Fig. 1. This fiber cable equation [1] is given by equation (4) from Laplace transformation with general solution. The output waveform comes from the inverse Laplace transform. The membrane propagation is simulated as shown in Fig. 4. For example, $\mathrm{R}$ is $1.59 / \mathrm{d}^{2}$ using resistivity $\rho 125[\Omega . m], C$ is $0.0296 \mathrm{~d}$, accordingly $\mathrm{RC}$ is $0.047 / \mathrm{d}$, where $\mathrm{d}$ is axon's diameter, $0.02 \mathrm{~mm}$. This signal is transmitted from cranial nerve and spinal cord to arm muscle to control the servomotor.

$$
\begin{aligned}
& \frac{\partial^{2} V(s)}{\partial x^{2}}=s R C V(s), \\
& V(s)=V_{m} \exp (-x \sqrt{s R C}), \\
& V=\frac{V_{m} x}{2 t^{3 / 2}} \sqrt{R C / \pi} \exp \left(-\frac{x^{2} R C}{4 t}\right)
\end{aligned}
$$

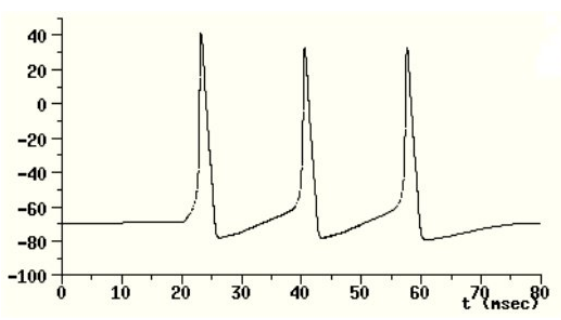

Fig. 4. Simulated membrane propagation.

\section{B. Robotic Arm Control Implementation}

Robotic arm implementation is based on the surface EMG to acquire the membrane action potential as shown in Fig. 5 of control system implementation. An electrode is silver/silver choloride $(\mathrm{Ag} / \mathrm{AgCl})$ because of its low half-cell potential of approximately $220 \mathrm{mV}$ and its ease of manufacturability. $\mathrm{Ag} / \mathrm{AgCl}$ electrodes are nonpolarized electrodes. They 
allow current to pass across the interface between the electrolyte and the electrode. Nonpolarized electrodes are better than polarized electrodes in terms of their rejection of motion artifacts and their response to defibrillation current

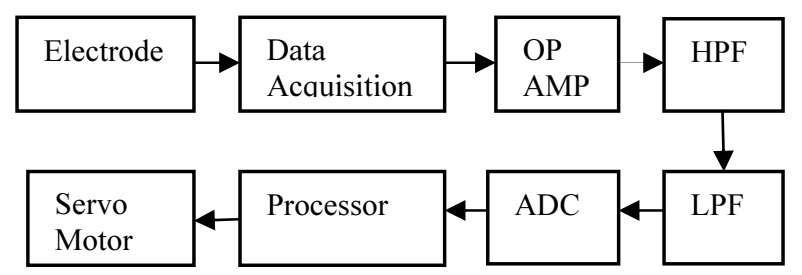

Fig. 5. System implementation blocks.

Both motion artifacts and defibrillation events can charge up the capacitance from the electrolyte and electrode interface. The $\mathrm{AgCl}$ layer lowers the impedance of the electrode. This is important at low frequencies near dc, where EMG measurements are taken. Skin to electrode impedances at $10 \mathrm{~Hz}$ using silver/silver chloride electrodes, with the skin properly prepared, are typically about $5 \mathrm{k} \Omega$. A simplified equivalent circuit for the electrode is shown in Fig. 6. Analysis of the circuit reveals the signals acquisition problem due to the $\mathrm{RC}$ components. Resistor $\mathrm{R}_{1}$ and capacitor $\mathrm{C}_{1}$ are due to the effects at the electrode/cell interface and are frequency dependent. These values fall off to a negligible point at a rate of $1 /(2 \pi f)^{2}$ and are considerably lower than $R_{2}$ and $C_{2}$.

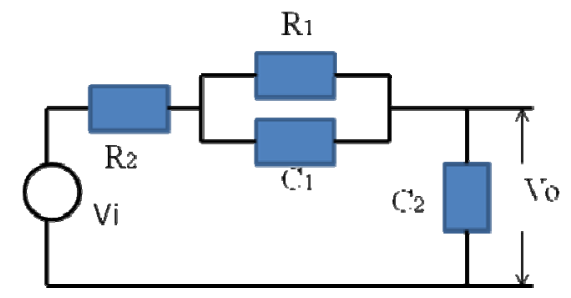

Fig. 6. Electrode equivalent circuit.

There are several important properties to consider in a pre-amplifier: High common mode rejection ratio, Very high input impedance, Short distance to the signal source and Strong DC signal suppression. The common mode rejection ratio provides an index on the extent to which common signal components are attenuated from the signal. As such it is desirable to have the highest common mode rejection (CMR) possible. At the moment the best CMRR that can be realistically achieved with current technology is about over $100(\mathrm{~dB} /$ Octave)

The signals had been amplified to recordable levels. The final step was to ensure that there would only be frequency content between $20[\mathrm{~Hz}]$ and 420 [Hz]. To do this, the amplified signal was passed through a secondorder highpass filter implemented with 20 [Hz] cutoff frequency and a second-order lowpass filter with a 420 [Hz] cutoff frequency. Second order filters were selected as a first order filter did not have sharp enough frequency cutoffs. Highpass filtering is necessary because movement artifacts are comprised of low frequency components. Lowpass filtering is desirable to remove high frequency components to avoid signal aliasing.

Measuring and accurately representing the EMG signal depends on the properties of the electrodes and their interaction with the skin, amplifier design, and the conversion and subsequent storage of the EMG signal from analog to digital conversion. The quality of the measured EMG is often described by the ratio between the measured EMG signal and unwanted noise contributions from the environment. The goal is to maximize the amplitude of the signal while minimizing the noise. Assuming that the amplifier design and process of $\mathrm{A} / \mathrm{D}$ conversion exceed acceptable, the signal to noise ratio is determined almost exclusively by the 3 electrodes.

Processor controls the servomotor for a robotic arm from ADC. Typical servomotors are very useful in different kinds of small robotic applications because of compact and inexpensive. RC servomotor includes a built-in DC motor, gear mechanism, position feedback control loop (potentiometer), and motor drive circuit. Servos refer to an error sensing feedback control which is used to correct the performance of a system. The pulse width modulation (PWM) technique makes reference to the position of the output shaft of the motor. The pulse width is converted into an equivalent voltage that is compared with that of signal from the potentiometer. That has DC motors equipped with a servo mechanism for precise control of angular position, be moved to a desired angular position by sending PWM signals. The motors usually have a rotation limit from $90^{\circ}$ to $180^{\circ}$ or 360 . The rotation is restricted in between the fixed angles.

\section{EXPERIMENT AND RESULTS}

The experiments are taken by signal acquisition to 8 persons to control vertical arm, ADC 10bits and sampling frequency $1000[\mathrm{~Hz}]$ for EMG, and by servo control from EMG between human and robot arm. The biceps signal is detected strongly in bending arm for the arm control system. The reference electrode is positioned at $2 \mathrm{~cm}$ down from the biceps and the ground electrode is placed at $3 \mathrm{~cm}$ outside to control the servomotor as shown in Fig. 7 (a) and (b). The spectral characteristics of the EMG signal will reflect the properties of a fixed set of muscle fibers in the region of the electrode. Also, the frequency spectrum of the EMG signal will be independent of any trigonometric factor that would provide an erroneous estimate of the conduction velocity. The resultant value of the conduction velocity affects the EMG signal by altering the temporal characteristics of the EMG signal, and consequently its frequency spectrum as shown in Fig. 7 (c) and (d).

A servo angles are controlled by impulse, and the centering is important. A servo precision positioning 
makes them ideal for robotics, since servos are self contained with control loop circuitry, drive circuits, servo position and speed control. Servos actively move to hold the position.

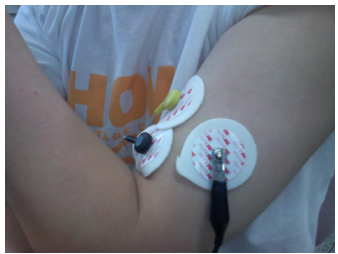

(a) Electrodes position

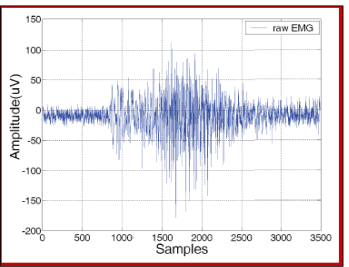

(c) Detected signal

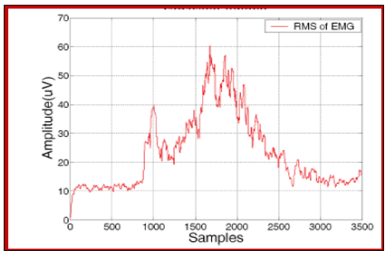

(e) RMS signal

Fig. 7. EMG electrodes and servo, and time and frequency outputs.

If a servo is instructed to move in the clockwise position and an external force is present and pushing against the servo, the servo resist being moved out of that position or continue to try and move to the instructed position, even if the servo arm is incorrectly placed on the motor shaft, until power off. It is for the reason that servo output arm or wheel should be placed into the neutral position before installation. The Table I shows the vertical arm angles and impulse intervals at the averages.

TABLE I

\section{SERVOMOTOR IMPULSE TIMES AND ANGLES}

\begin{tabular}{|c|c|c|c|c|c|c|c|c|c|c|}
\hline interval & 1 & 2 & 3 & 4 & 5 & 6 & 7 & 8 & 9 & 10 \\
\hline time & 180 & 160 & 140 & 120 & 100 & 90 & 80 & 70 & 60 & 20 \\
\hline angle & 0.7 & 0.9 & 1.0 & 1.2 & 1.4 & 1.5 & 1.6 & 1.7 & 1.8 & 2.0 \\
\hline
\end{tabular}

The error ranges of robotic arm control at the average are measured by 8 persons in repeating every 10 times. They take a rest for 5 minutes to minimize the muscle fatigue. The measured levels are taken by the minimum and maximum placed by $50^{\circ}=0$ and $180^{\circ}=100$ because individuals EMG amplitude of the waveforms are different. That results in 5\% lower of error as shown in the Fig. 8, which bottom curve is mean error, top curve indicates maximum error and mid curve shows minimum error.

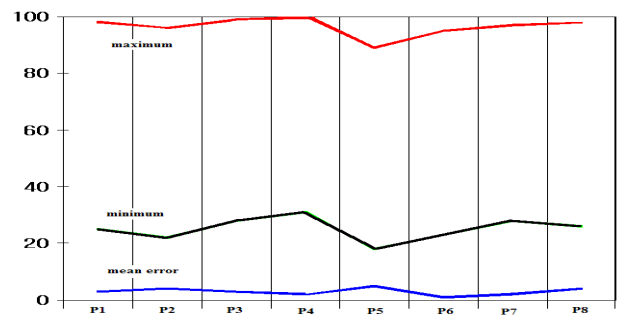

Fig. 8. Mean error ranges for practical control.

\section{CONCLUSIONS}

An implementation for surface EMG network analysis to vertical control system of robotic arm is presented in this paper. The system is realized to derive from the fine EMG transmembrane signals of human arm muscles are detected by several surface electrodes, high performance amplifier and filtering, converting analog to digital data and driving a servomotor for spontaneous robotic arm control. The system is experimented by monitoring multiple steps vertical control angle corresponding to voluntary arm movements. The experimental results are that the vertical arm control level is measured to around 2 degrees and mean error is lower $5 \%$. The system will be improved to be $3 \mathrm{D}$ controller for the handicapped in the future.

\section{REFERENCES}

[1] Sid Deutsch and Alice Deutsch, Understanding the Nervous System an Engineering Perspective, IEEE Press, 1993.

[2] Joseph D. Bronzino, The Biomedical Engineering Handbook, IEEE Press, 1995.

[3] Joseph J. Carr and John M. Brown, Introduction to Biomedical Equipment Technology, $3^{\text {rd }}$ edition, Prentice Hall, 1998.

[4] Scott day, Important Factors in Surface EMG Measurement, Bortec biomedical Ltd.

[5] Stepen Jung, John Meklenburg abd Sean Patrick, Surface Electromyogram Simulator for Myoelectric Prosthesis Testing, Worcester Polytechnic Institute, 2010

[6] Soke Hwan Kim, Kwang Ryol Ryu, and Chang Wu Hur, "A Study on Signal Transformation Neuron NO", Proc. Fall of KIMICS, 2001.

[7] Panagiotis K. Artemiadis and Kostas J. Kyriakopoulos, "An EMGbsed Robot Control Scheme Robust to Time-Varying EMG Signal features", IEEE Information technology in Biomedicine, vol.14, no. 3,2010 .

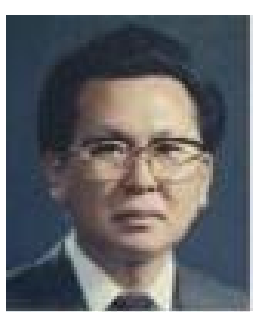

Kwang Ryol Ryu Received the PhD. Degree from Kyunghee University in 1988, and is currently a professor at the Dept. of Electronic Engineering at Mokwon University, worked as a visiting Professor at the Dept. of Neurological Surgery at University of Pittsburgh Medical Center in USA in 2006 2008. His areas of research include Digital Signal Processing, Imaging and Vision, and Bio-Medical Electronics. 